\title{
CHALLENGING COVID-19*
}

Dear Reader,

Coronaviruses are a large family of zoonotic viruses, that is, the virus is transmitted from animals to humans. Although several common human coronaviruses are known, and usually lead upon infection to mild to moderate upper-respiratory tract illnesses, some coronaviruses cause epidemics. Severe Acute Respratory Syndrome (SARS-CoV) erupted in February 2003 following transmission from civet cats to humans. Middle East Respiratory Syndrome (MERS-CoV) surfaced in April 2012 following transmission from dromedary camels to humans. However, both these epidemics pale into insignificance in the context of the havoc currently being caused by an hitherto unknown coronavirus, now named as COVID-19 and which is first assumed to have started mid-December 2019 in Wuhan, China. It is strongly suspected that COVID-19 was transmitted from bats to humans.

Coronavirus pathogenesis is a highly complex process and the much-needed details in hostpathogen interaction in COVID-19 infection are still being understood. Deriving from the lessons learnt from containing SARS and MERS, as well as zika, ebola and chikungunya, the Global Research Collaboration for Infectious Disease-Preparedness, GloPID-R, aims to contain the spread of disease and focus on transmission and diagnosis, management measures at human-animal interface, clinical characterisation and management of the disease, research and development for candidate therapeutics and vaccines, ethical considerations for research as well as integration of social sciences into the outbreak responses.

The COVID-19 virus uses the same cell entry receptor, angiotensin converting enzyme 2 (ACE 2), as the SARS-CoV. Different strategies are being investigated to fight COVID-19. One strategy is to repurpose treatments for HIV, hepatitis $B$, hepatitis $C$ and influenza. Another strategy is to focus on approved drugs or those that have passed clinical trials. Developent of vaccines, monoclonal antibodies, oligonucleotide-based therapies, peptides, interferon therapies as well as small molecule drugs are under way, but usually it takes years before they can be commonly used.

The global cooperation during this crisis of the COVID-19 epidemic, with WHO in the lead, is remarkable and heartening. The genome sequences of the virus were deposited as soon as they were generated in the open access database, Global Initiative on Sharing All Influenza Data, GISAID. This has led to the design of primers for PCR tests at the Charité, Berlin. Two one-step quantitative realtime reverse transcriptase PCR tests have been developed at Hong Kong University. A fluorescence-based RT-PCR kit has been developed by BGI Group, Shenzhen.

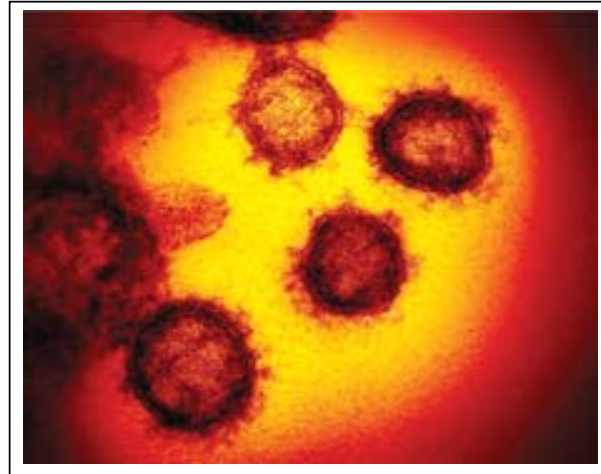

Picture: COVID-19 virus emerging from the surface of human cells. Image credit: NIAID$R M L$ Using this kit, a single lab can test up to 10,000 samples per day. An assay platform and a two-part test targeting $E$ and $F$ genes have been developed by Altona Diagnostics in Hamburg. Web-based dashboard to visualize and track reported cases of COVID-19 in realtime has been developed by John Hopkins University. The Vaccine Research Center of the US NIH communicated the COVID-19 viral sequence to collaborators at Moderna, which is developing a $m R N A$ vaccine under a programme funded by the Coalition for Epidemic Preparedness Innovations (CEPI). CEPI is also collaborating with CureVac, with GSK and with the University of Queensland in other similar projects. Regeneron Pharmaceuticals and the US Department of Health and Human Services are working 
towards development of new anti-coronavirus drugs. GeoVax USA and BravoVax China as well as Takis and Evvivax (both Italy) are focussing on vaccine development. iBio and CC-Pharming are working on a plant-derived vaccine. Gilead Sciences are in talks with USA and China for possible use of remdesivir, an investigational drug with efficacy in preclinical models of SARS and MERS coronaviruses. Clinical trials of the antivirals lopinavir and ritonavir have been initiated. Some research groups in China are investigating traditional Chinese medicines to treat COVID-19.

The COVID-19 vaccine candidate being developed jointly by the Serum Institute of India and Codagenix USA is currently in the pre-cinical/animal testing phase.

Artificial intelligence $(A I)$ is being used in different ways to find possible treatment for COVID-19. Benevolent $A$ I and Imperial College London are employing Al to find an already approved drug that might limit the virus's ability to infect people. Benevolent Al's algorithms connect molecular structure data to biomedical information about relevant receptors and diseases to find potential drug targets. The group adapted its search to newly available information about COVID-19 and focussed on the enzyme adapter-associated protein kinase. The Janus kinase inhibitor baricitinib emerges as a potential treatment for COVID-19, acting by reducing the ability of the virus to infect lung cells. Insilico Medicine Hong Kong has published six molecular structures for the key protein target of COVID-19.

The global consequences of the COVID-19 epidemic are a matter of concern to one and all. Western biotech companies depend significantly on Chinese CROs, such as WuXi AppTec. The province Hubei, where Wuhan is located, manufactures and exports 153 drugs. It is hoped that China and the world at large will be able to recover from the aftermath of COVID-19 soon.

*Dedicated to the late ophthalmologist Dr Li Wenliang

https://doi.org/10.53879/id.57.02.p0005

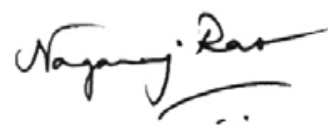

Dr. Nagaraj N. Rao Associate Editor Indian Drugs

\section{About The Associate Editor}

Dr. Nagaraj Narayan Rao obtained Bachelor's degrees in Science (Chemistry) and in the Technology of Pharmaceuticals and Fine Chemicals from the University of Mumbai. After working with Colgate-Palmolive (India) for two years as a laboratory chemist, he obtained his doctorate in science with magna cum laude from the University of Tuebingen, Germany, under the guidance of Prof. Dr. H. J. Roth. He carried out post-doctoral research at the Institute of Biotechnology of the Research Center Juelich, Germany. He was a member of the Editorial Board for the first official German-language version of the European Pharmacopoeia. He was a visiting scientist at Juelich and a visiting faculty at the Institute of Chemical Technology Mumbai from 1993 to 2007 in the field of bioprocess technology. He has authored several original research articles, a patent, review articles and book chapters in the fields of pharmaceuticals, biotechnology, brewery and surface coatings. He was Chief Editor of the "Transactions of the MFAl" for a few years. He contributes a monthly 'Report from India' to a leading German technical journal since fourteen years and is a distinguished alumnus of the Research Center Juelich.

Dr. Rao is co-founder of the RRR group of small and medium enterprises, manufacturing organic fine chemicals, formulations for surface coating technologies and fertilizers, process sensors and process units for life sciences, brewery and chemical process industries, as well as representing select overseas companies for cell culture media, bulk drugs and used chemical equipment and plants.

4

If you would like to comment on the editorial please write to us at publications@idmaindia.com 